\title{
Self-Similar Solidification of Binary Alloys
}

\author{
D.V. Alexandrov, A.A. Ivanov and A.P. Malygin \\ Department of Mathematical Physics, Urals State University \\ Ekaterinburg 620083, Russian Federation
}

\begin{abstract}
The self-similar crystallization process of a binary mixture from a cooled boundary is studied on the basis of two models with a planar front and mushy layer. Approximate analytical solutions of the process describing the solidification with a mushy layer are found. The theory under consideration is in good agreement with experiments carried out by Huppert and Worster for ice growing from aqueous salt solutions.
\end{abstract}

PACS numbers: 44.05.+e, 81.30.Fb

\section{Introduction}

Mathematical descriptions of crystallization processes play very important role in crystal growth, engineering, oceanography and metallurgy. The transition amorphous-to-crystalline in the materials for optical phase-change memories is of great practical importance. The mathematical models allow predicting many properties of solids produced by melt cooling. If the liquid is an alloy (a mixture of two or more components) its crystallization process completely differs from the solidification of a pure liquid. In particular, various distributions of impurity in the solid phase lead to different mechanical and physical properties of ingots. This phenomenon arises due to the impurity displacement into the melt by the moving front of solidification. If the impurity displacement is rather intensive, the constitutional supercooling originates ahead of the planar solid-liquid interface and, generally speaking, the two-phase zone (mushy region) appears. Solid nuclei in the form of newly born crystals may evolve in this zone. The development of this system reduces the supercooling and leads to formation of a new stable solidification mode characterized by the presence of a mushy layer that separates the pure solid and melt regions. Heat and mass transfer in the mushy layer has a major effect on the properties of the solid materials thus produced, which is also responsible for the marked interest in the study of solidification of binary melts accompanied by formation of the aforementioned transition layer.

Mathematical descriptions of solidification scenario are complicated not only by nonlinearities of heat and mass transfer equations but also by the need to apply boundary conditions at solid-liquid interfaces which are evolving with time and whose positions must be determined as a part of the solution. A full set of thermodynamic equations for a mushy layer is developed and a much-reduced set of them is solved approximately in Ref. [1] for the constrained growth of a binary alloy. However, constrained growth, in which the interfaces are supposed to advance at a prescribed constant velocity, is applicable to industrial crystal pulling (the Czochralski growth), but not to the solidification of castings nor to many natural systems where growth can proceed at a rate dependent on time and, in particular, at a rate inversely proportional to the square root of time. The aim of this paper is to develop the theory where the interfaces propagate at rates dependent on time.

\section{Self-similar solidification with a planar front}

Let us consider a unidirectional solidification process of a binary melt along $z$-axis from a cooled boundary experimentally studied in Ref. [2]. Let us consider a semi-infinite region $z>0$ filled with liquid which initially has uniform composition $C=c_{0}$ and temperature $T_{\infty}$. We traditionally neglect the solute transport in the solid phase and, following Ref. [3], ignore the effects of gravity and imagine that the plane $z=0$ forms the lower, horizontal boundary of the domain. The temperature of the cooled boundary is maintained at a value $T=T_{B}$ lower than the initial liquidus temperature. The temperature $(T)$ and concentration $(C)$ fields in the solid and liquid phases are described by the classical heat and mass transfer equations

$$
\begin{aligned}
\rho_{\mathrm{S}} C_{p \mathrm{~S}} \frac{\partial T}{\partial t} & =k_{\mathrm{S}} \frac{\partial^{2} T}{\partial z^{2}}, \quad 0<z<h(t), \\
\rho_{\mathrm{L}} C_{p \mathrm{~L}} \frac{\partial T}{\partial t} & =k_{\mathrm{L}} \frac{\partial^{2} T}{\partial z^{2}}, \quad \frac{\partial C}{\partial t}=D \frac{\partial^{2} C}{\partial z^{2}}, \\
0 & <z<h(t),
\end{aligned}
$$

where $\rho$ is the density, $C_{p}$ is the specific heat, $k$ is the thermal conductivity, $D$ is the diffusion coefficient and $z=h(t)$ is the frontal coordinate dependent on time $t$ (subscripts S and L designate the solid and liquid phases, respectively); all of the transfer coefficients are assumed to be constant in each phase. In the case of unidirectional regime with a planar front, nothing depends on the spatial coordinate directed perpendicular to the solidification direction. The boundary conditions imposed at the solid wall $z=0$ and far from the phase transition interface can be expressed in the form

$$
\begin{aligned}
& T=T_{B}, \quad z=0, \\
& C \rightarrow c_{0}, \quad T \rightarrow T_{\infty}, \quad z \rightarrow \infty .
\end{aligned}
$$

Further, we consider the case when the front is close to 
equilibrium, that is

$T(h, t)=-\Gamma C\left(h^{+}, t\right), \quad z=h(t)$.

Heat and solute must be conserved at the front

$$
\begin{gathered}
\rho_{\mathrm{S}} L \frac{\mathrm{d} h}{\mathrm{~d} t}=k_{\mathrm{S}}\left(\frac{\partial T}{\partial z}\right)_{z=h^{-}}-k_{\mathrm{L}}\left(\frac{\partial T}{\partial z}\right)_{z=h^{+}}, \\
z=h(t), \\
C\left(h^{+}, t\right) \frac{\mathrm{d} h}{\mathrm{~d} t}+D\left(\frac{\partial C}{\partial z}\right)_{z=h^{+}}=0, \quad z=h(t),
\end{gathered}
$$

where $L$ stands for the latent heat parameter. The aforementioned model (1)-(7) admits a similarity solution with variable $\eta$ and interface coordinate $h(t)$ of the form

$$
\eta=z / \sqrt{4 D t}, \quad h(t)=\lambda \sqrt{4 D t},
$$

where the parabolic growth rate constant $\lambda$ is a part of the solution.

Omitting mathematical manipulations, we express the solution of problem (1)-(7) in terms of self-similar variables (8); the result is

$$
\begin{aligned}
& T(\eta)=T_{B}+\frac{\left(T_{h}-T_{B}\right) \operatorname{erf}\left(\varepsilon_{\mathrm{S}} \eta\right)}{\operatorname{erf}\left(\varepsilon_{\mathrm{S}} \lambda\right)}, \quad \eta<\lambda, \\
& T(\eta)=T_{\infty}+\frac{\left(T_{h}-T_{\infty}\right) \operatorname{erfc}\left(\varepsilon_{\mathrm{L}} \eta\right)}{\operatorname{erfc}\left(\varepsilon_{\mathrm{L}} \lambda\right)}, \quad \eta>\lambda, \\
& C(\eta)=c_{0}+\frac{\left(C_{h}-c_{0}\right) \operatorname{erfc}(\eta)}{\operatorname{erfc}(\lambda)}, \quad \eta>\lambda,
\end{aligned}
$$

where $\varepsilon_{\mathrm{S}}=\sqrt{D C_{p \mathrm{~S}} \rho_{\mathrm{S}} / k_{\mathrm{S}}}, \varepsilon_{\mathrm{L}}=\sqrt{D C_{p \mathrm{~L}} \rho_{\mathrm{L}} / k_{\mathrm{l}}}, T_{h}$ and $C_{h}$ stand for the temperature and concentration at the solidification front $\eta=\lambda$. Three unknowns can be found from the boundary conditions (5)-(7). Substitution of distributions (9)-(11) into (5)-(7) gives

$$
\begin{gathered}
T_{h}=-\Gamma C_{h}, \quad C_{h}-c_{0}=C_{f i}(\lambda)=\frac{c_{0} F(\lambda)}{1-F(\lambda)}, \\
\Gamma C_{f i}(\lambda)\left[\frac{\beta}{F\left(\varepsilon_{\mathrm{L}} \lambda\right)}+\frac{1}{G\left(\varepsilon_{\mathrm{S}} \lambda\right)}\right]= \\
\frac{T_{1}}{G\left(\varepsilon_{\mathrm{S}} \lambda\right)}-\frac{\beta T_{0}}{F\left(\varepsilon_{\mathrm{L}} \lambda\right)}-\frac{L}{C_{p \mathrm{~S}}},
\end{gathered}
$$

where

$$
\begin{aligned}
& \beta=\frac{\rho_{\mathrm{L}} C_{p \mathrm{~L}}}{\rho_{\mathrm{S}} C_{p \mathrm{~S}}}, \quad F(x)=\sqrt{\pi} x \exp \left(x^{2}\right) \operatorname{erfc}(x), \\
& G(x)=\sqrt{\pi} x \exp \left(x^{2}\right) \operatorname{erf}(x) .
\end{aligned}
$$

Equation (13) determines the parabolic growth rate constant $\lambda$. The driving temperature differences $T_{1}=$ $-\Gamma c_{0}-T_{B}, T_{0}=T_{\infty}+\Gamma c_{0}$ and graphs of $\lambda$ versus $T_{1}$, $T_{0}$, and $c_{0}$ are shown in many figures in Ref. [3].

It is noteworthy that the temperature ahead of the planar solidification front can fall below the local liquidus temperature. This phenomenon is called "constitutional supercooling". This supercooling arises if the concentration gradient exceeds the temperature one at the front. Mathematically, this condition can be written in the form

$$
\left(\frac{\partial T}{\partial z}\right)_{z=h^{+}}<-\Gamma\left(\frac{\partial C}{\partial z}\right)_{z=h^{+}} .
$$

Substituting (10) and (11) into (14) and taking into ac- count (12), we rewrite inequality (14) for the self-similar case as

$$
C_{f i}(\lambda)>C_{i}(\lambda)=\frac{\left(T_{0} / \Gamma\right) \varepsilon_{\mathrm{L}}^{2} F(\lambda)}{F\left(\varepsilon_{\mathrm{L}} \lambda\right)-\varepsilon_{\mathrm{L}}^{2} F(\lambda)} .
$$

If the left- and right-hand sides of (15) become identical, we have a supercooling onset. Therefore if (15) holds true the aforementioned description of the solidification scenario with a planar front becomes inapplicable and we shall use a mushy layer model.

\section{Self-similar solidification with equilibrium mushy layer}

We analyze the solidification of a binary melt with a mushy layer, in which heterogeneous inclusions of the new phase grow in such a manner that this layer is virtually totally desupercooled. In this case, a mushy layer may be treated as independent of the precise morphology of the growing solid phase. The mush is also treated as a continuum, and its physical properties are taken to be functions of the local volume fraction of solid $\varphi$. Heat and mass transfer are described by heat conduction and diffusion equations in the mushy layer $a(t)<z<b(t)$ $(a(t)$ and $b(t)$ stand for the solid-mush and mush-liquid boundaries)

$$
\begin{aligned}
& \left(\rho C_{p}\right)_{\mathrm{m}} \frac{\partial T}{\partial t}=\frac{\partial}{\partial z}\left(k_{\mathrm{m}} \frac{\partial T}{\partial z}\right)+\rho_{\mathrm{S}} L \frac{\partial \varphi}{\partial t}, \\
& \frac{\partial}{\partial t}(\chi C)=D \frac{\partial}{\partial z}\left(\chi \frac{\partial C}{\partial z}\right), \\
& T=-\Gamma C, \quad \chi=1-\varphi,
\end{aligned}
$$

where subscript $\mathrm{m}$ denotes properties of the mushy layer. The thermal properties of the mushy layer are assumed to be volume-fraction-weighted averages of the properties of the individual phases so that [3]:

$$
\begin{aligned}
& k_{\mathrm{m}}(\varphi)=k_{\mathrm{L}}(1-\varphi)+k_{\mathrm{S}} \varphi, \\
& \left(\rho C_{p}\right)_{\mathrm{m}}=\rho_{\mathrm{L}} C_{p \mathrm{~L}}(1-\varphi)+\rho_{\mathrm{S}} C_{p \mathrm{~S}} \varphi .
\end{aligned}
$$

The heat and mass flux conditions imposed at the boundaries $z=a(t)$ and $z=b(t)$ have the form

$$
\begin{aligned}
& \rho_{\mathrm{S}} L \chi_{a} \frac{\mathrm{d} a}{\mathrm{~d} t}=k_{\mathrm{S}}\left(\frac{\partial T}{\partial z}\right)_{z=a^{-}}-k_{\mathrm{m}}\left(\frac{\partial T}{\partial z}\right)_{z=a^{+}} \\
& C_{a} \chi_{a} \frac{\mathrm{d} a}{\mathrm{~d} t}=-D \chi_{a}\left(\frac{\partial C}{\partial z}\right)_{z=a^{+}}, \\
& \rho_{\mathrm{S}} L\left(1-\chi_{b}\right) \frac{\mathrm{d} b}{\mathrm{~d} t}=k_{\mathrm{m}}\left(\frac{\partial T}{\partial z}\right)_{z=b^{-}}-k_{\mathrm{l}}\left(\frac{\partial T}{\partial z}\right)_{z=b^{+}} \\
& \left(\frac{\partial T}{\partial z}\right)_{z=b^{+}}=-\Gamma\left(\frac{\partial C}{\partial z}\right)_{z=b^{+}}, \\
& C_{b}\left(1-\chi_{b}\right) \frac{\mathrm{d} b}{\mathrm{~d} t}=D \chi_{b}\left(\frac{\partial C}{\partial z}\right)_{z=b^{-}}-D\left(\frac{\partial C}{\partial z}\right)_{z=b^{+}} .
\end{aligned}
$$

The governing Eqs. (16) and (17) and the boundary conditions (18) admit similarity solutions with the same 
similarity variable $\eta$ and interface positions of the form

$$
a(t)=\lambda_{a} \sqrt{4 D t}, \quad b(t)=\lambda_{b} \sqrt{4 D t}
$$

where $\lambda_{a}$ and $\lambda_{b}$ are the parabolic growth rate constants (mushy layer coordinates in the self-similar solidification mode).

Taking into account that relaxation times of the solute concentration field is several orders of magnitude higher than the thermal relaxation time, the temperature field in the mushy layer can be written as a linear function of coordinate $z$ (see, among others [4-7]). Furthermore, taking into account that the diffusion field in the mushy layer is practically frozen and the mass transport is caused by the impurity displacement into the liquid phase we use a linear temperature approximation and the Scheil equation for the impurity distribution in the mushy layer [4-7]. Thus, instead of (16), we have

$$
T(\eta)=T_{3}+T_{2} \eta, \quad C(\eta)=\frac{A}{1-\varphi(\eta)} .
$$

Substituting $\varphi=\varphi_{a}$ at $\eta=\eta_{a}$ and $\varphi=\varphi_{b}$ at $\eta=\eta_{b}$ into (20) and keeping in mind (17), we get

$$
\begin{aligned}
& A=-\frac{1}{\Gamma}\left(1-\varphi_{b}\right)\left(T_{3}+T_{2} \lambda_{b}\right), \\
& T_{3}=\frac{T_{2}\left[\lambda_{b}\left(1-\varphi_{b}\right)-\lambda_{a}\left(1-\varphi_{a}\right)\right]}{\varphi_{b}-\varphi_{a}} .
\end{aligned}
$$

Now, using (17), (19) and (20), we rewrite the boundary conditions (18) for the determination of $\varphi_{a}, \varphi_{b}, \lambda_{a}, \lambda_{b}$ and $T_{2}$ in the form

$$
\begin{aligned}
& -\frac{1}{\Gamma}\left(T_{3}+T_{2} \lambda_{b}\right)=c_{0}+C_{i}\left(\lambda_{b}\right), \\
& \left\{\frac{\varepsilon_{\mathrm{S}}^{2}\left(1-\varphi_{b}\right)}{\alpha}+\frac{k_{\mathrm{m}}\left(\varphi_{b}\right)}{k_{\mathrm{S}}}\left[c_{0}+C_{i}\left(\lambda_{b}\right)\right]\right. \\
& \left.\quad-\frac{C_{i}\left(\lambda_{b}\right)}{F\left(\lambda_{b}\right)}\right\} \varphi_{b}=0, \quad \alpha=\frac{\Gamma C_{p \mathrm{~S}}}{L}, \\
& \frac{T_{2}}{\Gamma}=2 \lambda_{b}\left[\frac{k_{l}}{k_{\mathrm{m}}\left(\varphi_{b}\right)} \frac{C_{i}\left(\lambda_{b}\right)}{F\left(\lambda_{b}\right)}+\frac{\varepsilon_{\mathrm{S}}^{2}}{\alpha} \frac{k_{\mathrm{S}}}{k_{\mathrm{m}}\left(\lambda_{b}\right)} \varphi_{b}\right], \\
& {\left[2 \lambda_{a} \frac{\varepsilon_{\mathrm{S}}^{2}}{\alpha}\left(1-\varphi_{a}\right)+\frac{k_{\mathrm{m}}\left(\varphi_{a}\right)}{k_{\mathrm{S}}} \frac{T_{2}}{\Gamma}\right] G\left(\varepsilon_{\mathrm{S}} \lambda_{a}\right)} \\
& +\frac{2 \lambda_{a} \varepsilon_{\mathrm{S}}^{2}}{\Gamma}\left[T_{B}-\left(T_{3}+T_{2} \lambda_{a}\right)\right]=0, \\
& {\left[T_{2}+2 \lambda_{a}\left(T_{3}+T_{2} \lambda_{a}\right)\right]\left(1-\varphi_{a}\right)=0 .}
\end{aligned}
$$

Generally speaking, four regimes (1) $\varphi_{b}=0, \varphi_{a}=1$; (2) $\varphi_{b}=0, \varphi_{a} \neq 1$; (3) $\varphi_{b} \neq 0, \varphi_{a}=1$, and (4) $\varphi_{b} \neq 0$, $\varphi_{a} \neq 1$ can be considered as possible solidification scenarios. However two cases $\varphi_{b}=0, \varphi_{a}=1$ and $\varphi_{b} \neq 0$, $\varphi_{a}=1$ lead to $T_{3}+T_{2} \lambda_{b}=0$ (see Eq. (21)). The latter suggests that the impurity concentration $c_{0}+C_{i}\left(\lambda_{b}\right)$ at the boundary mushy layer-liquid phase goes to zero (see Eq. (22)). Therefore, these unphysical cases will be omitted from our consideration.

Further, omitting tedious and cumbersome mathematical manipulations following from (21)-(26) let us now rewrite the final formulae for two possible regimes: case $(2) \varphi_{b}=0, \varphi_{a} \neq 1$ :

$$
\begin{aligned}
& T_{2}\left(\lambda_{b}\right)=\frac{2 \lambda_{b} \Gamma C_{i}\left(\lambda_{b}\right)}{F\left(\lambda_{b}\right)}, \\
& \varphi_{a}\left(\lambda_{b}\right)=2 \lambda_{a}\left(\lambda_{b}\right)\left[\lambda_{b}-\lambda_{a}\left(\lambda_{b}\right)\right], \\
& \lambda_{a}\left(\lambda_{b}\right)=\frac{\Sigma\left(\lambda_{b}\right)-\sqrt{\Sigma^{2}\left(\lambda_{b}\right)-8 \lambda_{b}^{2} C_{i}^{2}\left(\lambda_{b}\right)}}{4 \lambda_{b} C_{i}\left(\lambda_{b}\right)}, \\
& \left\{\frac{2 \lambda_{a}\left(\lambda_{b}\right) \varepsilon_{\mathrm{S}}^{2}}{\alpha}\left[1-\varphi_{a}\left(\lambda_{b}\right)\right]\right. \\
& \left.\quad+2\left[\Lambda+(1-\Lambda) \varphi_{a}\left(\lambda_{b}\right)\right] \Omega\left(\lambda_{b}\right)\right\} G\left(\varepsilon_{\mathrm{S}} \lambda_{a}\left(\lambda_{b}\right)\right) \\
& \quad+2 \varepsilon_{\mathrm{S}}^{2}\left[\frac{\lambda_{a}\left(\lambda_{b}\right) T_{B}}{\Gamma}+\Omega\left(\lambda_{b}\right)\right]=0 ;
\end{aligned}
$$

case $(4) \varphi_{b} \neq 0, \varphi_{a} \neq 1$ :

$$
\begin{aligned}
& T_{2}\left(\lambda_{b}\right)=\frac{2 \lambda_{b} \Gamma}{\Lambda+(1-\Lambda) \varphi_{b}\left(\lambda_{b}\right)} \\
& \quad \times\left[\frac{\Lambda C_{i}\left(\lambda_{b}\right)}{F\left(\lambda_{b}\right)}+\frac{\varepsilon_{\mathrm{S}}^{2} \varphi_{b}\left(\lambda_{b}\right)}{\alpha}\right], \\
& \varphi_{b}\left(\lambda_{b}\right)= \\
& \quad \frac{C_{i}\left(\lambda_{b}\right) / F\left(\lambda_{b}\right)-\varepsilon_{\mathrm{S}}^{2} / \alpha-\Lambda\left[c_{0}+C_{i}\left(\lambda_{b}\right)\right]}{(1-\Lambda)\left[c_{0}+C_{i}\left(\lambda_{b}\right)\right]-\varepsilon_{\mathrm{S}}^{2} / \alpha}, \\
& \lambda_{a}\left(\lambda_{b}\right)=\frac{\Xi\left(\lambda_{b}\right)-\sqrt{\Xi^{2}\left(\lambda_{b}\right)-2}}{2}, \\
& \varphi_{a}\left(\lambda_{b}\right)=\varphi_{b}\left(\lambda_{b}\right) \\
& +2 \lambda_{a}\left(\lambda_{b}\right)\left[\lambda_{b}-\lambda_{a}\left(\lambda_{b}\right)\right]\left[1-\varphi_{b}\left(\lambda_{b}\right)\right], \\
& \left.\left\{\begin{array}{l}
2 \lambda_{a}\left(\lambda_{b}\right) \varepsilon_{\mathrm{S}}^{2} \\
\alpha
\end{array}\right] \varphi_{a}\left(\lambda_{b}\right)\right] \\
& \left.+\left[\Lambda+(1-\Lambda) \varphi_{a}\left(\lambda_{b}\right)\right] \frac{T_{2}\left(\lambda_{b}\right)}{\Gamma}\right\} G\left(\varepsilon_{\mathrm{S}} \lambda_{a}\left(\lambda_{b}\right)\right) \\
& +\frac{\varepsilon_{\mathrm{S}}^{2}}{\Gamma}\left[2 \lambda_{a}\left(\lambda_{b}\right) T_{B}+T_{2}\left(\lambda_{b}\right)\right],
\end{aligned}
$$

where

$$
\begin{aligned}
& \Sigma\left(\lambda_{b}\right)=2 \lambda_{b}^{2} C_{i}\left(\lambda_{b}\right)+F\left(\lambda_{b}\right)\left[c_{0}+C_{i}\left(\lambda_{b}\right)\right], \\
& \Lambda=\frac{k_{\mathrm{L}}}{k_{\mathrm{S}}}, \\
& \Xi\left(\lambda_{b}\right)=\lambda_{b}+\left[c_{0}+C_{i}\left(\lambda_{b}\right)\right] \Gamma / T_{2}\left(\lambda_{b}\right), \\
& \Omega\left(\lambda_{b}\right)=\lambda_{b} \frac{C_{i}\left(\lambda_{b}\right)}{F\left(\lambda_{b}\right)},
\end{aligned}
$$

and formulae (29) and (34) represent transcendental equations for the determination of $\lambda_{b}$. Thus, we have two different analytical solutions $((27)-(29)$, case $(2)$ and (30)-(34), case (4)) describing the self-similar solidification scenario. 


\section{Concluding remarks}

Figures 1-3 illustrate analytical solution in accordance with two possible cases (2) and (4). Region I corresponds

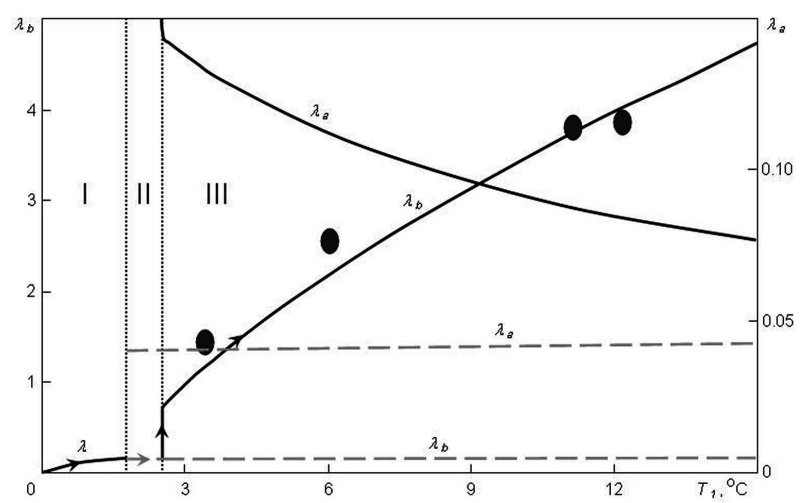

Fig. 1. Parabolic growth rate constants as functions of $T_{1}=-\Gamma c_{0}-T_{B}$. Solid lines correspond to the frontal (region I) and mushy layer (region III, case (2)) solidification scenarios. Dashed lines illustrate the formal solution in accordance with case (4). Region II describes intermediate (transitional) regime between regions I and III. Vertical dotted lines show the boundaries between different solidification modes. Physical parameters of sodium nitrate $\mathrm{NaNO}_{3}$ are given in Ref. [3]. The circles are data from the experiments of Huppert and Worster [2]. $T_{\infty}=15^{\circ} \mathrm{C}, c_{0}=14$ (measures the weight per cent of $\mathrm{NaNO}_{3}$ ).

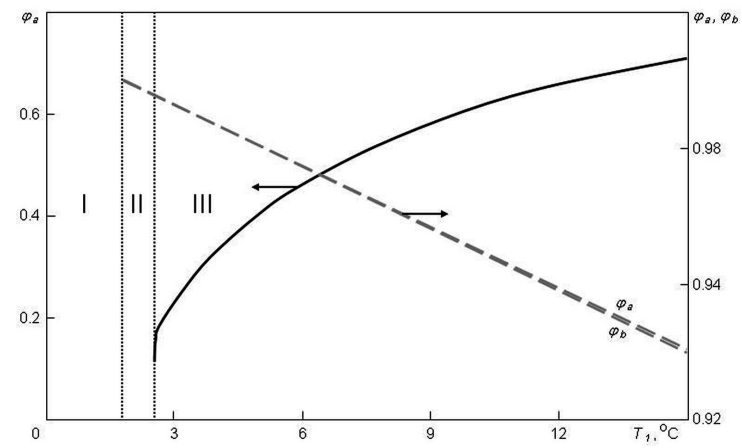

Fig. 2. Solid fractions for case (2) (solid line) and case (4) (dashed lines) as functions of temperature $T_{1}$. Dashed lines close together exist in regions II and III. However, in region III, only solid lines represent physical solutions whereas dashed lines show the formal solution. Physical parameters of sodium nitrate $\mathrm{NaNO}_{3}$ are given in Ref. [3].

to the planar solidification front model (expressions (12) and (13)) up to the temperature difference $T_{1}$ when the left- and right-hand sides of inequality (15) become equal. Immediately afterwards inequality (15) holds true and the classical description of the regime with a planar front becomes inapplicable. In this case, strictly speaking, we have solutions (30)-(34), case (4). These solutions

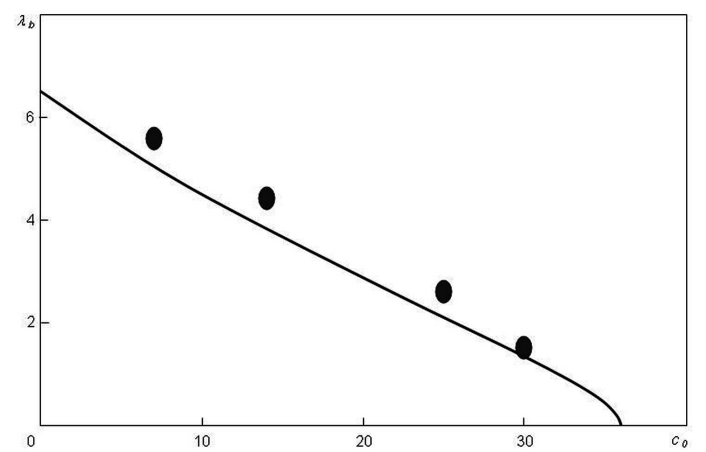

Fig. 3. The parabolic growth rate constant $\lambda_{b}$ as a function of $c_{0}$. Physical parameters of sodium nitrate $\mathrm{NaNO}_{3}$ are given in Ref. [3]. The circles are data from the experiments of Huppert and Worster [2]. $T_{\infty}=15^{\circ} \mathrm{C}, T_{B}=-17^{\circ} \mathrm{C}$.

are continuous in terms of $\lambda=\lambda_{b}$ and discontinuous in terms of $\lambda_{a}, \varphi_{a}$ and $\varphi_{b}$ at the boundary between regions I and II (when $T_{1}$ agrees with the constitutional supercooling condition $C_{f i}=C_{i}$ ). In other words, at this point $\lambda_{a}, \varphi_{a}$ and $\varphi_{b}$ abruptly appear in such a manner that the solid fractions lie so close to unity that a mushy layer influence is negligible in practical situations. However, region II is responsible for the instability evolution [3], which leads to the appearance of another solidification mode (expressions (27)-(29), case (2)) in the presence of a mushy layer. Mathematically, these solutions appear at the boundary between regions II and III and, apparently, they are stable contrary to the second branch of solutions (case (4)), which conceptually corresponds to the instability evolution of small perturbations for the planar front in region II. Thus, expressions (27)-(29) hold true in region III. Also, the latter is confirmed by experimental data, which demonstrate the mushy layer evolution in accordance with case (2). What is more, regions II and III were found on the basis of numerical solution of the problem under consideration [3]. The question now arises of whether there is a single solution in region II. As this metastable region reflects the effect of constitutional supercooling, its existence is connected with nucleation and growth of newly born solid crystals in the supercooled liquid matrix. The latter, however, is out of the equilibrium theory under consideration where such effects are ignored. Let us especially emphasize in conclusion that the parabolic growth rate constants $\lambda_{a}$ and $\lambda_{b}$ (solid curves, Fig. 1, case (2)) within the framework of our approximate analytical solutions approach to a joint point at the boundary between regions II and III.

\section{Acknowledgments}

This work was made possible due to the financial support of grants Nos. 08-01-00298, 09-08-00844, 07-0396069 Ural, 07-01-96091 Ural (Russian Foundation for Basic Research), MD-4563.2008.2. (President Grant) and 2.1.1/2571 (Ministry of Education). 


\section{References}

[1] R.N. Hills, D.E. Loper, P.H. Roberts, Quantum J. Appl. Math. 36, 505 (1983).

[2] H.E. Huppert, M.G. Worster, Nature 314, 703 (1985).

[3] M.G. Worster, J. Fluid Mech. 167, 481 (1986).

[4] D.V. Alexandrov, A.P. Malygin, I.V. Alexandrova, Ann. Glaciol. 44, 118 (2006).
[5] D.V. Alexandrov, I.G. Nizovtseva, Dokl. Earth Sci. 419, 359 (2008).

[6] D.V. Alexandrov, I.G. Nizovtseva, A.P. Malygin, H.-N. Huang, D. Lee, J. Phys., Condens. Matter 20, 114105 (2008).

[7] D.V. Alexandrov, D.L. Aseev, I.G. Nizovtseva, H.-N. Huang, D. Lee, Int. J. Heat Mass Transfer 50, 3616 (2007). 